\title{
Top-down attention and consciousness: comment on Cohen et al.
}

\author{
Naotsugu Tsuchiya ${ }^{1,2}$, Ned Block ${ }^{3}$, and Christof Koch ${ }^{4,5}$ \\ ${ }^{1}$ School of Psychology and Psychiatry, Monash University, Clayton, Victoria 3800, Australia \\ 2 Japan Science and Technology Agency, Tokyo 102-0076, Japan \\ ${ }^{3}$ Department of Philosophy, New York University, 5 Washington Place, New York, NY 10003, USA \\ ${ }^{4}$ Division of Biology, California Institute of Technology, Pasadena, CA 91125, USA \\ ${ }^{5}$ Allen Institute for Brain Science, Seattle, WA 98103, USA
}

Cohen and colleagues masterfully summarize the psychophysical and neurophysiological evidence pertaining to the distinction between visual attention and visual consciousness, concluding that attention is necessary but not sufficient for consciousness [1]. We disagree with their view for the following reasons.

In our own work, we have been cautious to claim only that one can become conscious of an attribute of some class of objects in the near absence of top-down, endogenous attentional amplification [2,3]. Given the close relationship between stimulus strength and bottom-up, exogenous attention, we do not think it is possible to dissociate bottomup attention from consciousness.

To our mind, the function of both forms of attention is to resolve ambiguity due to large, overlapping receptive fields and the presence of competing objects within cluttered natural scenes. The brain accomplishes this mainly by amplifying the neuronal representation of the attended attribute at the expense of unattended stimuli. These topdown attentional signals originate in frontal and parietal cortical regions, and feed back into earlier sensory corticothalamic stages of processing. However, for detection or coarse discrimination of an isolated object, top-down attention does not play a significant role [4]. There is no computational reason and no evidence for the claim that top-down attention is required to become conscious of simple, isolated objects, such as a single oriented bar.

With psychophysical manipulation alone, diffuse topdown attention to a stimulus cannot be excluded. However, optogenetics has provided methods to transiently, delicately, and reversibly turn molecularly identified neuronal cell types or even just their connections off and on again for time-scales lasting from milliseconds to hours. Although such invasive techniques are off-limits in humans, they are being routinely implemented in mice $[5,6]$ and, to a much more limited extent, in monkeys [7-9].

In the next few years, it will become possible to reversibly turn off feedback from higher cortical regions to visual cortex, without interfering with the forward flow of information. We would argue that under these conditions, top-down attention will have been effectively locked out of visual cortex. It will then become possible to address the question of whether the animals can still respond in a behaviorally meaningful manner to such a non-attended stimulus. Such experiments require sophisticated behavioral controls and very selective molecular tools, as well as large-scale observatories to record the activity of large numbers of genetically identified cell types. This is the aim of the ongoing project MindScope at the Allen Institute for Brain Science [10].

Lastly, the authors are overly restrictive in their supposition that, if any one of four distinct ways of withdrawing attention can eliminate conscious perception, then consciousness requires attention. None of these four tasks are 'pure' attentional manipulations: they change processing in a variety of ways, depending on the type of stimuli and behavioral demands. We are not claiming that every way of withdrawing attention leaves consciousness intact, but rather that the two are distinct neurobiological processes that can, under particular conditions, be dissociated.

\section{References}

1 Cohen, M.A. et al. (2012) The attentional requirements of consciousness. Trends Cogn. Sci. 16, 411-417

2 Koch, C. and Tsuchiya, N. (2007) Attention and consciousness: two distinct brain processes. Trends Cogn. Sci. 11, 16-22

3 van Boxtel, J.J. et al. (2010) Consciousness and attention: on sufficiency and necessity. Front. Psychol. 1, 217

4 Lee, D.K. et al. (1999) Attention activates winner-take-all competition among visual filters. Nat. Neurosci. 2, 375-381

5 Deisseroth, K. (2011) Optogenetics. Nat. Methods 8, 26-29

6 Yizhar, O. et al. (2011) Optogenetics in neural systems. Neuron 71, 9-34

7 Han, X. et al. (2009) Millisecond-timescale optical control of neural dynamics in the nonhuman primate brain. Neuron 62, 191-198

8 Diester, I. et al. (2011) An optogenetic toolbox designed for primates. Nat. Neurosci. 14, 387-397

9 Gerits, A. et al. (2012) Optogenetically induced behavioral and functional network changes in primates. Curr. Biol. 22, 1722-1726

10 Koch, C. and Reid, R.C. (2012) Neuroscience: observatories of the mind. Nature 483, 397-398

1364-6613/\$ - see front matter @ 2012 Elsevier Ltd. All rights reserved. http://dx.doi.org/10.1016/j.tics.2012.09.004 Trends in Cognitive Sciences, November 2012, Vol. 16, No. 11 